\title{
Reflexiones sobre las características constitutivas de la gestión integral*
}

\author{
Reflections on the constituent characteristics \\ of the integral management
}

Recibido: 27 de agosto de 2009

Revisado: 30 de septiembre de 2009

Aceptado: 09 de octubre de 2009

\section{Fabio Tejada Losada* \\ Guillermo Peña Guarín \\ Miembros del grupo de investigación "Gestión Integral" \\ del Convenio USTA-ICONTEC, Bogotá, Colombia}

\section{Resumen}

En este artículo se presenta la forma como se interpreta el concepto de "gestión integral" en el contexto de la Maestría en Calidad y Gestión Integral. Para ello se analiza en primer lugar el concepto de "la calidad", planteándose una visión ampliada del mismo que excede la relación tradicional entre un cliente y su proveedor. Posteriormente se analiza la configuración interna de una organización a partir de cuatro elemen-

* Artículo de reflexión sobre temáticas originales y asociadas a investigación.

** Correspondencia: Fabio Tejada Losada. Magíster en calidad y gestión integral. Docente investigador de tiempo completo del convenio de la Universidad Santo Tomás e ICONTEC. fatelo@etb.net.co.

Guillermo Peña Guarín. Magíster en calidad y gestión integral. Auditor Líder ISO 9001 de ICONTEC. Actualmente se desempeña como Coordinador académico y Docente Investigador del Convenio Universidad Santo Tomás - ICONTEC. gpena@icontec.org guillerpegu@yahoo.com tos diferenciados pero articulados entre sí: la estructura del negocio, el direccionamiento estratégico, la cultura de la organización y los sistemas de gestión; éstos últimos deberían estar integrados con base en la compatibilidad de sus elementos.

Pero la gestión integral incluye también elementos externos a la organización relacionados, en el enfoque de esta Maestría, con los tres componentes del desarrollo sostenible: el crecimiento económico, la protección del medio ambiente y la cohesión social; de modo que la gestión integral debe abarcar la compleja integración de los elementos internos y externos que permiten la sostenibilidad organizacional.

Palabras clave: calidad. gestión integral, sostenibilidad de las organizaciones.

Abstract
This article presents the form as is interpreted the
concept of " integral management " in the context of
the Mastery in Quality and Integral Management. We
examine first the concept of " the quality ", appearing 
an extended vision of the same one that exceeds the traditional relation between a client and its supplier. Later the internal configuration of an organization is analyzed from four elements differentiated but articulated: the structure of the business, the strategic management, organizational culture and management systems; the latter mentioned should be integrated based on the compatibility of its elements. But the integral management includes also external elements to the organization related, in the approach of this Mastery, to three components of the sustainable development: the economic growth, the protection of the environment and the social cohesion; so that the integral management must include the complex integration of the internal and external elements that enable the organizational sustainability.

Key words: integral management, organizational sustainability, quality.

\section{Introducción}

El convenio académico entre la Universidad Santo Tomás y el Instituto Colombiano de Normas Técnicas y Certificación (ICONTEC) orienta desde hace tres años el programa de Maestría en Calidad y Gestión Integral; el propósito general de esta maestría está encaminado a:

\section{[...] el desarrollo, la validación y la profundización de conoci- miento en el campo de la gestión de la calidad y a su integra- ción con otros sistemas de gestión existentes y futuros, para favorecer el sostenimiento en el tiempo y el mejoramiento continuo de las organizaciones y para facilitar la inserción de la responsabilidad social en aquellas organizaciones que han implementado o desean implementar sistemas de gestión, de modo que éstas contribuyan a mejorar la cohesión social en el país (Convenio, USTA-ICONTEC, 2006).}

Esta intencionalidad amplia y desafiante requiere que se precise la forma como se interpreta el concepto de la "gestión integral" en el marco de dicha maestría, para guiar la actuación de la comunidad académica y para diferenciar éste de otros programas, dándole la pertinencia que demandan las circunstancias actuales de las organizaciones y de la sociedad colombiana.

Para plantear la interpretación de la gestión integral es necesario efectuar primero un breve análisis del concepto "calidad", referente básico dentro del campo de estudio y práctica de la Maestría.

\section{Entendiendo "la calidad"}

La calidad es tema obligado de reflexión en el contexto de la Maestría en Calidad y Gestión Integral y sigue siendo un referente ineludible cuando se trata de proponer orientaciones que contribuyan a la sostenibilidad y competitividad de las organizaciones. Sin embargo, no hay una definición universalmente aceptada para la idea de "la calidad" y son múltiples las acepciones que ésta ha tenido a través de la historia.

Las dificultades para definirla pueden generar equívocos, sobre todo cuando no se hacen distinciones cuidadosas sobre el nivel de análisis al que nos referimos (un producto, un proceso, una organización, un mercado o un sistema); estas dificultades conceptuales se hacen más notorias al momento de proponer métodos para evaluar dicha calidad.

Al respecto, Camisón, Cruz y González (2006) postulan que las diferentes acepciones del término calidad se han ido "construyendo como respuestas a cambios en los problemas empresariales, sustentando diferentes orientaciones para la Gestión de la Calidad (p.146)". Los autores condensan esos diferentes enfoques conceptuales en la clasificación que se presenta en la Tabla 1. 
Tabla 1. Conceptos de calidad. Fuente: Camisón et al., (2006).

\begin{tabular}{|c|c|c|c|}
\hline Autores & Enfoque & Acento diferencial & Desarrollo \\
\hline Platón & Excelencia & $\begin{array}{l}\text { Calidad absoluta } \\
\text { (producto) }\end{array}$ & $\begin{array}{l}\text { Excelencia como calidad absoluta, "lo mejor" } \\
\text { Asimilación con el concepto de "lujo" } \\
\text { Analogía con la calidad de diseño }\end{array}$ \\
\hline $\begin{array}{l}\text { Shewhart } \\
\text { Crosby }\end{array}$ & $\begin{array}{l}\text { Técnico: conformidad con } \\
\text { especificaciones }\end{array}$ & $\begin{array}{l}\text { Calidad comprobada/controlada } \\
\text { (procesos) }\end{array}$ & $\begin{array}{l}\text { Establecer especificaciones } \\
\text { Medir la calidad por la aproximación real a los estándares } \\
\text { Énfasis en la calidad de conformidad. Cero defectos. }\end{array}$ \\
\hline $\begin{array}{l}\text { Deming } \\
\text { Taguchi }\end{array}$ & $\begin{array}{l}\text { Estadístico: pérdidas } \\
\text { mínimas para la sociedad, } \\
\text { reduciendo la variabilidad y } \\
\text { mejorando estándares }\end{array}$ & $\begin{array}{c}\text { Calidad generada } \\
\text { (producto y procesos) }\end{array}$ & $\begin{array}{l}\text { La calidad es inseparable de la eficacia económica } \\
\text { Un grado predecible de uniformidad y fiabilidad a bajo coste } \\
\text { La calidad exige disminuir la variabilidad de las características del pro- } \\
\text { ducto alrededor de los estándares y su mejora permanente } \\
\text { Optimizar la calidad de diseño para mejorar la calidad de conformidad }\end{array}$ \\
\hline $\begin{array}{l}\text { Feigenbaum } \\
\text { Juran } \\
\text { Ishikawa }\end{array}$ & Aptitud para el uso & $\begin{array}{l}\text { Calidad planificada } \\
\quad \text { (sistema) }\end{array}$ & $\begin{array}{l}\text { Traducir las necesidades de los clientes en las especificaciones } \\
\text { La calidad se mide por lograr la aptitud deseada por el cliente } \\
\text { Énfasis tanto en la calidad de diseño como de conformidad }\end{array}$ \\
\hline $\begin{array}{l}\text { Parasuraman } \\
\text { Berry } \\
\text { Zeithaml } \\
\end{array}$ & $\begin{array}{l}\text { Satisfacción de las expectati- } \\
\text { vas de los clientes }\end{array}$ & $\begin{array}{l}\text { Calidad satisfecha } \\
\quad \text { (servicio) }\end{array}$ & $\begin{array}{l}\text { Alcanzar o superar las expectativas de los clientes } \\
\text { Énfasis en la calidad de servicio }\end{array}$ \\
\hline $\begin{array}{c}\text { Evans } \\
\text { [Procter \& } \\
\text { Gamble] }\end{array}$ & Calidad total & $\begin{array}{l}\text { Calidad gestionada } \\
\text { (empresa y su sistema de valor) }\end{array}$ & $\begin{array}{l}\text { Calidad significa crear valor para los grupos de interés } \\
\text { Énfasis en la calidad en toda la cadena y el sistema de valor }\end{array}$ \\
\hline
\end{tabular}

Como se puede observar, en las anteriores definiciones de la calidad se encuentra una amplia gama de enfoques que varían desde la apreciación de la calidad como algo sensible y subjetivo (percepción - satisfacción) con énfasis en atributos que pueden ser arbitrarios o contingentes, hasta su consideración en términos más objetivos y racionales (excelencia - crear valor) que hacen énfasis en el carácter de la calidad como algo universal y necesario, énfasis que le hace perder sus connotaciones relativas y tiende a convertirla en un concepto absoluto.

En el contexto de las organizaciones, estos diversos conceptos de la calidad se pueden interpretar y expresar desde una perspectiva interna o desde una perspectiva externa a ellas.

El control estadístico de los procesos, la generación de cero defectos y el énfasis en determinar las especificaciones distinguen a la perspectiva interna, en la cual subyace la premisa de que si los productos (bienes 0 servicios) se elaboran asegurando su uniformidad (sin variaciones), en forma eficiente y buscando la excelencia, encontrarán acogida en el mercado; por otra parte, la idea de calidad como valor agregado, la satisfacción de las expectativas de los clientes y la aptitud para el uso denotan la perspectiva externa, la cual corresponde más a mercados competitivos en los que el cliente puede escoger entre diversas alternativas y el énfasis radica en la eficacia. ${ }^{1}$

En la definición de la calidad propuesta por las normas de la serie ISO 9000, referente obligado para los sistemas de gestión de la calidad, se busca equilibrar

1 Una aproximación a una definición más global de la calidad fue planteada por la Asociación Española para la Calidad; se enfoca en hacer coincidir las expectativas de los clientes (calidad esperada) con los resultados que efectivamente es capaz de entregar la organización (calidad realizada) y éstas con las especificaciones que la organización planificó (calidad diseñada); la calidad global podría evaluarse en función de los resultados alcanzados en reducir las brechas entre estas tres "dimensiones de la calidad". 
las perspectivas interna y externa, al establecer que la calidad es el "grado en el que un conjunto de características inherentes cumple con los requisitos" (ICONTEC, 2005); se aclara en las notas de la definición que el término "inherentes" se usa en contraposición a "asignadas", es decir, que las características a las cuáles hace referencia son propias y permanentes de aquello a lo que se le determina la calidad (que puede ser un producto tangible, un servicio, un proceso o un sistema), mientras que los requisitos son externos, y pueden ser generados por las diferentes "partes interesadas"2.

A la coincidencia plena entre los requisitos y las características inherentes se le denominaría buena calidad, pero si el grado de cumplimiento es menor se puede hacer mención a una regular, baja o pobre calidad; por el contrario, cuando las características inherentes superan a los requisitos, la calidad se podría denominar como superior 0 excelente.

Lograr las características inherentes implica que la organización hace uso de todas sus capacidades en la mejor forma posible (eficiencia) con el fin de cumplir con los requisitos de sus partes interesadas (eficacia).

Este enfoque de la calidad establecido por la norma ISO 9000:2005 puede ser demasiado abstracto ya que no precisa el sujeto al cual se le aplica (un bien, un servicio, un proceso, un sistema o una organización), pero incluye un aspecto clave en la concepción de la gestión integral porque considera que en una organización las responsabilidades en cuanto a la calidad no se limitan a sus clientes, sino que deben considerar a otras partes interesadas. Bajo este enfoque se rompe

2 Estas "partes interesadas" pueden incluir a los clientes, propietarios, personal de la organización, proveedores o la sociedad, como lo enuncian las notas de las definiciones de "parte interesada", NTC-ISO 9001:2005. (numeral 3.3.7) y de "requisito" NTC-ISO 9001:2005. (numeral 3.1.2). la relación cliente - proveedor (productor - usuario; entidad - beneficiario), una premisa que siempre había estado asociada a las definiciones de la calidad.

Otros autores, al momento de definir la calidad, hacen énfasis en el precio que un consumidor está dispuesto a pagar por un bien o por un servicio, es decir, al valor que tiene para el cliente, un aspecto en el cual no es explícita la definición de ISO 9001:2005. Ishikawa plantea al respecto que "no podemos definir la calidad sin tener en cuenta el precio. Esto cobra importancia al planear y diseñar la calidad. No puede haber control de calidad que haga caso omiso del precio, las utilidades y el control de costos" (Ishikawa, 1986, p.41).

El planteamiento de Ishikawa es certero porque se enfoca en los dos aspectos clave de la calidad: el interno (eficiencia y productividad) y el externo (eficacia y precio), con lo cual se entra a considerar el concepto de valor para el cliente como la "relación entre calidad y precio".

El análisis acerca de la definición de calidad se hace más complejo cuando la organización se observa como un todo y no de manera fragmentada, y se aprecia que las organizaciones están integradas a un entorno social y tienen responsabilidades no sólo para sus clientes sino también, como lo menciona la norma ISO 9000:2005, para otras partes interesadas.

Por ello el concepto de la calidad se amplía para incluir a los clientes y a otras partes interesadas; en tal sentido, Camisón plantea como definición de calidad "la creación global de valor por la organización para todos sus grupos de interés clave" y precisa que este enfoque de "calidad total" constituye un "nuevo concepto de valor como el grado de satisfacción de las expectativas de todos los grupos de interés de la organización, incluyendo a los accionistas, mejor, más rápida y más eficientemente que la competencia" (Camisón, 2006, p. 196). 
En el marco de la Maestría en Calidad y Gestión Integral las actividades de enseñanza - aprendizaje, la investigación formativa, aplicada e institucional y la proyección social se refieren a este concepto amplio de calidad que incluye los aspectos relativos al cliente, a los productos y a los procesos productivos, pero que además se extiende a la calidad de vida dentro de las organizaciones y a la calidad de las relaciones entre la organización y las comunidades implicadas en sus actividades productivas

Dentro de este enfoque es evidente que la orientación de las organizaciones que buscan mejorar sus niveles de eficiencia y eficacia para incrementar su competitividad, afianzándose para ello en sistemas de gestión, demanda una comprensión más amplia de la organización y de sus diferentes componentes, de tal manera que se interpreten como verdaderos sistemas productivos, que para alcanzar sus resultados en entornos cada vez más amplios, inciertos y, por lo tanto, más complejos, requieren de mayor capacidad de dirección para asegurar su sostenibilidad.

\section{De la administración a la gestión integral}

Indudablemente, la conducción de las organizaciones y de los negocios hasta hace unos años, cuando las condiciones del mercado lo permitían, podía en gran medida asegurarse con los elementos que se desprendín de las prácticas administrativas tradicionales. Eran esos tiempos de economías cerradas y proteccionistas en que los empresarios y encargados de dirigir las empresas se concentraban prioritariamente en prácticas internas que, en tales circunstancias de mercado, les garantizaban buena parte de los resultados esperados.

Tales prácticas estaban sujetas a la orientación de la administración, que buscaba asegurar la aplicación de procedimientos a través de los cuales se fijaban y comunicaban los programas y se regularizaba el desarrollo de las actividades, de tal manera que permitiera su contraste con los objetivos y planes definidos. Esta forma de concebir las empresas centraba su actividad en la operación, en los costos y los gastos ligados fundamentalmente a los procesos de producción o para la ejecución del servicio.

No podemos desconocer que en escenarios pasados, como en los presentes, las organizaciones de cualquier naturaleza centralizan sus energías en alcanzar resultados que les permitan su consolidación y sustenten sus futuras actividades. En este enfoque los resultados económicos y los aspectos ligados con los recursos económicos ocupan gran parte de la atención de quienes dirigen las empresas. Y esto, en nuestro concepto, no está mal. No se puede negar que tratándose de una empresa privada con ánimo de lucro, por ejemplo, los inversionistas, quienes asumen un riesgo y colocan recursos financieros o de otra índole en un negocio, esperan el retorno de sus capitales multiplicados, pues de lo contrario no apostarían su capital económico o industrial en empresas que no tienen dentro de sus planes alcanzar este tipo de objetivos.

Puede seguirse asegurando que buena parte del "qué" de las organizaciones, referido a la definición de los propósitos y resultados esperados en ellas, continúa siendo bastante claro a pesar de los vertiginosos cambios que se siguen presentando en tramos de tiempo cada vez más cortos, y que demandan de las organizaciones y de quienes las dirigen, renovados esfuerzos para asegurar tales resultados en entornos cada vez más complejos y turbulentos (Tejada, 2009).

El mundo de las organizaciones ha cambiado sustancialmente. Existen multiplicidad de factores que explican las profundas transformaciones que han ocurrido en las últimas décadas. Los avances de la tecnología en general, el acceso en mayor escala a la información y a 
la producción de conocimiento práctico y aplicable en los diferentes ámbitos organizacionales y económicos, los enormes progresos en las tecnologías de la información y las comunicaciones - sólo por mencionar unos pocos -. Cambios que han traído consigo y generado a partir de sus interacciones una verdadera revolución en los sistemas de producción y sus relaciones.

Otro gran impacto, aunque menos reciente, pero no por esto menos importante, se originó como resultado de acuerdos económicos entre las naciones cuando se avanzó con mayor decisión en fortalecer y flexibilizar la movilidad de productos y servicios a través de regulaciones comerciales entre diferentes naciones del orbe, que abrieron los mercados y posibilitaron el acceso a una mayor gama de productos y servicios que en el pasado, en el campo local, se encontraban restringidos a unos pocos. La consecuencia inmediata y rápida fue que se pasó de un mercado de productores-oferentes a un mercado de demandantes.

En Colombia, esto se materializó en los años noventa del siglo pasado con la apertura económica y sigue avanzando hoy, siendo su motor de impulso la globalización ${ }^{3}$ y sus diferentes manifestaciones, lo que genera un profundo impacto en los mercados y las fuerzas que los regulan.

La dinámica que se ha presentado en el mundo de los negocios a partir de los cambios nos ha mostrado desde la realidad de su comportamiento, que el concepto tradicional de administración y la forma como se conducían las organizaciones ${ }^{4}$ para asegurar resultados en el

3 Creciente gravitación de los procesos financieros, económicos, políticos, sociales y culturales de alcance mundial sobre los de carácter regional, nacional y local. (CEPAL, 2002)

4 Organizaciones que se debían orientar en economías cerradas, con mercados cautivos y protegidas, en no pocas ocasiones con fuertes barreras arancelarias que, no obstante la incertidumbre, se desempeñaban en ambientes más controlables, donde el foco de la administración se centraba más en los aspectos operativos que mediano y largo plazo ha dejado de ser suficiente para responder apropiadamente a los nuevos retos y realidades, producto de los grandes avances de la humanidad y sus interacciones económicas y sociales en todos los campos.

En este sentido, diversas investigaciones realizadas sobre la evolución que ha tenido la forma de pensar y concebir la organización muestran que desde finales del siglo XIX hasta la actualidad se han presentado drásticos cambios en su concepción y en los modelos que buscan explicar la forma más efectiva para su conducción y gobierno.

A nuestro juicio, uno de ellos se produce cuando los psicólogos sociales Robert Katz y Daniel Kahn presentan un trabajo más elaborado sobre las organizaciones de los que hasta la fecha se conocían ${ }^{5}$, en el que las determinan como un conjunto que se encuentra en alta interdependencia con el medio en el cual se desenvuelven y que tiene un propósito que le es común a todas las partes que integran ese conjunto (Dávila, 2001).

Este trabajo, que se conoce como la teoría sistémica de la organización, trae para los administradores grandes desafíos y nuevos retos. Si conducir una organización (que hasta tales momentos demandaba una alta concentración hacia su interior) ahora incluye el entorno como una variable que determina en grado sumo la orientación del sistema productivo, esto plantea una alta complejidad para su comprensión y adecuada orientación.

aseguraran el dominio de los gastos, de los costos de la mecánica operativa y el control administrativo.

5 Katz y Kahn desarrollan su teoría sistémica de la organización a partir de los estudios del biólogo Ludwig Von Bertalanffy (1930), cuyas primeras publicaciones se conocieron en 1950, y posteriormente, de Kennet Boulding (1956). 
Tal enfoque precisa de los administradores el dominio de todos los componentes internos de la organización, viéndolos ahora no como partes fraccionadas que apoyan un resultado final, sino como un conjunto que interactúa coherentemente en búsqueda de un propósito común y que, además, está en una estrecha correlación con el entorno en el cual se desempeña.

Esta forma de concebir la organización como un sistema ${ }^{6}$ productivo, también desafía y deja corto el concepto de administración tradicional con el cual se buscaba garantizar la conducción de las organizaciones y producir a partir de él claridad en su orientación y gobierno.

Asegurar resultados que permitan a la organización sostenibilidad, crecimiento y rentabilidad conciliando los planes y propósitos de corto, mediano y largo plazo, precisa de capacidades mayores o más elaboradas en quienes tienen a cargo dirigir los destinos de las empresas y, por consiguiente, conceptos y orientaciones renovados que expliquen y den luces sobre la conducción y el gobierno de organizaciones en escenarios cada vez más evolucionados y con mayores niveles de complejidad.

La organización visualizada hacia un enfoque de sistemas $^{7}$ es un imperativo que facilita entender los fenómenos y comportamientos que se dan en ella. Concebida en un concepto más evolucionado como un sistema abierto, en contraposición con la de sistema cerrado, permite entender que desarrollar capacidad

6 Sistema como un concepto amplio que rebasa los enfoques mecanicistas newtonianos con que se buscaban explicaciones para la construcción organizacional y sus impactos en los resultados

7 Las Norma NTC ISO 9000/2005 recoge el concepto sistémico organizacional llevándolo a nivel de principio: "enfoque sistémico para la gestión" que orienta y fortalece el desarrollo de sistemas de gestión de la calidad en todo tipo de organizaciones. Lo refuerza además con otro principio que está en relación directa con el anterior y es el de "enfoque en los procesos". para alcanzar los resultados planificados no sólo depende de administrar operaciones internas, sino también, y en gran medida, de lo que ocurre en el entorno de la empresa.

Supone entonces considerar las interrelaciones de la organización con su medio para dar orientación desde este enfoque a las actividades internas que permitan la generación de mayor orden, coherencia y concentración del quehacer de la empresa en el desarrollo de las actividades cruciales para el logro de los resultados importantes que se han planificado.

Como se ve, es una organización más distanciada de los conceptos mecanicistas ${ }^{8}$ que la determinaban como partes que de alguna forma alcanzaban sus resultados y apoyaban los de la organización. Tal orientación, la de sistema abierto, significa mayor cohesión y coherencia en los propósitos de la empresa, así como claridad en el enfoque de las operaciones y actividades, e invita más a la integración del conjunto organizacional. Es decir, una clara orientación hacia la integración con el entorno y a la integralidad de su conducción como un todo alrededor de propósitos comunes.

No es posible entonces pretender que la organización sea sostenible, rentable o pueda crecer sostenidamente si se concentra únicamente en los factores de su dinámica interna, desestimando el impacto que en ella tienen las permanentes modificaciones que ocurren en su entorno, en los mercados donde se desempeña.

Ante estas realidades, la gerencia y la gestión surgen entonces como conceptos más completos que involucran componentes renovados que superan -por su comprensión- aquéllos tradicionales ligados solamen-

8 El mecanicismo de Descartes y de Newton, que como idea filosófica pretende explicar la realidad, establece que ella debe ser entendida según los modelos proporcionados por la mecánica e interpretada sobre la base de la materia y el movimiento. 
te al concepto genérico de administración (Tejada, 2009) 9

La administración ha estado más orientada a la consecución, distribución y uso de recursos que permiten alcanzar resultados, así como a los controles de las actividades operativas y administrativas necesarias que buscan asegurar racionalidad para el logro de los objetivos.

La administración especializa y se enfoca en tal especialización. Los recursos que se manejan en las organizaciones -como son tan importantes desde el punto de vista administrativo- han fraccionado el orden organizacional alrededor de ellos al determinar tareas más o menos independientes ${ }^{10}$, tales como: recursos humanos, en actividades de personal; recursos financieros, en tareas financieras; recursos tecnológicos, en investigación y desarrollo; recursos de mercado, en actividades de marketing; recursos físicos, en tareas de producción; y el tiempo, en actividades de planificación. Los administradores se concentran en cada una de ellas, pero sin una articulación que interprete eficazmente el panorama general.

La gerencia, que en un espectro más amplio recoge la capacidad de la organización en interrelación constante con la generalidad y su entorno, supone la creación dinámica de escenarios propicios para que la empresa como un todo logre aprovechar toda su capacidad operativa y sus controles internos, para ajustarse cada

9 Existen otros puntos de vista sobre el concepto de administración, más ligados al "management", que pretenden mostrarlo como la ciencia o el arte que va desde la responsabilidad en la planificación y regulación económica y racional de una empresa, para alcanzar un objetivo o tarea determinada. Nosotros lo establecemos en el plano conceptual que, desde la práctica en organizaciones colombianas, se ha caracterizado por atender más las demandas mecanicistas de los negocios y allí se ha concentrado sin rasgos evolutivos que lo catapulten a niveles que involucren componentes que permitan mavor comprensión desde los ámbitos sistémicos.

10 Jan P. Sallenave hace relación al tema en su libro La gerencia integral (2004), cuando se refiere a los gerentes generalistas y a los especialistas, denominando a los primeros como integrales vez de manera más ágil a los constantes cambios en el entorno que pueden significar nuevas oportunidades en el mercado o problemas latentes que se pueden traducir en barreras insuperables para avanzar en la consecución de sus resultados

Es la gerencia, por lo tanto, la responsable de la definición de los ejes estratégicos de la organización que permitan enfocar hacia ellos el quehacer de la empresa, aprovechando todas las capacidades operativas, de tal manera que sea eficaz, pero, a la vez, con un alto grado de eficiencia que le permita fortalecer y sustentar mayores niveles de competitividad.

La gestión gerencial, por lo tanto, y en consideración a lo anterior, se debe enfocar en adoptar las decisiones críticas que determinan un conjunto de orientaciones y directrices que facilitan concentrar el trabajo en los factores clave de éxito empresarial, alrededor de procesos y actividades coherentemente interrelacionados que buscan un propósito común.

En este sentido, la gestión de la gerencia se encamina más a entender el entorno que impacta a la organización, para determinar los horizontes que guían el rumbo que debe seguir la organización de acuerdo a las renovadas capacidades y competencias empresariales, articulando las actividades del corto plazo con los propósitos del largo plazo, en pos de mayor competitividad que, finalmente, es lo que hace a las organizaciones sostenibles, rentables y con mayores posibilidades de crecimientos sostenidos.

En términos más llanos, se enfoca en una gestión integral que, como su nombre lo indica, involucre y armonice el medio externo con el medio interno, para generar claridad en la conducción y el gobierno de la organización hacia los horizontes definidos para la empresa. Escenario éste en el que el concepto de administración fortalece la concepción de integralidad, pero que por sí solo es insuficiente para producir toda la capacidad para 
responder a la complejidad en que se desenvuelven las organizaciones en un mundo más evolucionado en todos sus ámbitos.

\section{Gestión inteǵral interna}

La orientación moderna de las empresas de negocios y las organizaciones en general, no puede desconocer aquellos aspectos que son determinantes en su arquitectura ${ }^{11}$. No se puede desconocer que en las empresas de negocios, por ejemplo, la rentabilidad es uno de los aspectos críticos que demandan gran energía y concentración de recursos por parte de la gerencia; pero ella, la rentabilidad, no se logrará hasta que otros componentes críticos hayan sido considerados e involucrados para producir una gestión integral que permita avanzar con mayor equilibrio no sólo hacia la rentabilidad, sino también hacia el desarrollo de los demás aspectos que en la vida de las organizaciones contemporáneas son cruciales.

Como quedó ya advertido, las organizaciones deben entender que en el entorno surgen nuevas y permanentes demandas que hacen necesario focalizar la estrategia de la organización (o los métodos que utilice para producir tal focalización) en una mayor generación de sentido para toda la comunidad organizacional y de las partes interesadas.

Las organizaciones, cualquiera sea su naturaleza, deben por lo tanto fortalecer continuamente su capacidad para avanzar de manera eficaz en búsqueda de los resultados planificados. Hemos analizado, igualmente, que tales resultados serán factibles en la medida en que la gerencia se enfoque en orientar las operaciones de la empresa como un todo y no en la conducción

11 Concepto que implica una mayor elaboración en la concepción de la empresa, al considerar que en su construcción interviene un conjunto de componentes que, dentro de una visión sistémica, demanda articular los fenómenos externos con los aspectos internos de la organización dentro de una mirada holística e integradora. fragmentada, típica de la administración como concepto. Se trata de conducir más hacia la integración de sus componentes y una integralidad de la gestión que permita la generación de escenarios organizacionales comprensibles para todo el colectivo de la empresa.

En nuestro concepto, tal capacidad proviene de la claridad con que la gerencia ${ }^{12}$ sea capaz de articular sus componentes internos: direccionamiento estratégico, estructura del negocio, sistemas de gestión y cultura de la organización. La dinámica organizacional permanente debe tener como motor fundamental el conocimiento de los hombres y mujeres que la componen, con un enfoque de alta precisión y punto focal decidido hacia el mercado que deben entender, atender y en el cual pretenden hacerse sostenibles, rentables o crecer.

Revisemos brevemente los componentes planteados que debidamente articulados facilitan la gestión integral de las organizaciones.

\section{Estructura del negocio}

La definición superior de la organización como negocio, más allá de las estructuras tradicionales, es el plan y el modelo de negocios que la organización define y adopta para llegar al mercado y a los clientes. Conduce a la forma en que la organización genera valor para sus clientes desde la cadena de la realización del producto y el servicio. Es la definición clara y precisa de la cadena de generación de valor para un mercado o mercados determinados.

Esta estructura tiene una mayor comprensión y alcance que los ordenamientos organizacionales tradicionales que se demuestran a través de organigramas, estructuras organizacionales o cartas organizacionales.

12 Concebida la gerencia como el cuerpo gerencial que decide y conduce los destinos de la organización y no concentrada en una sola persona que la representa. 
La estructura de negocio tiene como propósito producir la claridad y enfoque correcto sobre qué es lo que la organización debe privilegiar alrededor de sus productos/servicios en clara interrelación con el mercado, de tal manera que pueda concentrarse en cómo va a llevar a esos mercados sus productos/servicios y que ellos sean reconocidos por atributos específicos que transmiten al cliente mayores niveles de satisfacción.

\section{Direccionamiento estratégico}

Hace relación a los horizontes que define la gerencia para dar sentido práctico a las acciones que la empresa desarrollará de manera crucial, de acuerdo con su estructura de negocio. Este direccionamiento permite a la organización avanzar y consolidarse en el mercado con claridad y concentración para alcanzar sus grandes propósitos, lleva a diferenciarse de la competencia a ser mejor que ella, a la vez que su operación es productiva y sostenible en el largo plazo.

Estos horizontes involucran la planificación, la organización, la dirección y control sobre el camino futuro de una empresa, así como la implementación de decisiones que mantienen a la organización en el curso correcto. Involucra componentes tales como visión, misión, principios, valores y la definición de estrategias generales, particulares o por unidades de negocio (según sea el tipo y tamaño de la organización, por ejemplo) que de manera práctica y útil permitan generar sentido en los diferentes actores de la organización, al determinar prioridades que lleven a focalizar conocimiento y recursos de manera ordenada en estrecha relación del medio externo con el medio interno, para producir una dinámica en la empresa que fortalezca la capacidad para alcanzar los resultados planificados en el corto, mediano y largo plazo.

El direccionamiento estratégico debe permitir una clara articulación de los niveles de pensamiento de la organización: el pensamiento estratégico con el pen- samiento de la ejecución y la coordinación, hasta el pensamiento de los niveles operativos.

Métodos de control como el Balanced score card son de alto impacto para asegurar que la organización se focaliza en las estrategias definidas y está concentrando las actividades operativas y recursos en los aspectos que estratégicamente son cruciales para el negocio.

\section{Sistemas de gestión integrados}

Estos sistemas hacen alusión a la determinación y adopción precisa de las partes (componentes) que conforman la organización como un todo y que se articulan alrededor de un propósito común.

Un sistema de gestión apoya el logro de los objetivos de la organización a través de estrategias y tácticas que involucran la optimización de procesos, el enfoque centrado en la gestión y el pensamiento disciplinado, que busca generar claridad para todos sus actores y equilibrio en sus diferentes componentes. Como sistema abierto tiene toda la capacidad de reconocer su medio interno en interacción permanente con el entorno del cual depende. En este contexto, los sistemas de gestión normalizados son un gran apoyo y han demostrado ser un paradigma válido $0^{13}$ para la gestión de las organizaciones.

Existen las normas de la serie ISO 9000, que facilitan el desarrollo y establecimiento de sistemas de gestión de la calidad; las normas ISO 14000, que orientan el establecimiento de sistemas de gestión medio ambiental; y las normas de la serie OHSAS 18000, que permiten el establecimiento de los sistemas de salud ocupacional y seguridad industrial ) por mencionar sólo algunas de las más aplicadas). Todas ellas proporcionan una exce-

13 Hernando Mariño N., en su escrito "Calidad: lecciones aprendidas" se refiere a esta expresión y la desarrolla con enfoque en los sistemas de gestión de la calidad 
lente ayuda para determinar y fortalecer sistemas de gestión en las organizaciones.

Por supuesto que no son los únicos sistemas que permiten gestionar hacia propósitos específicos en las empresas. Existen otros frentes de acción empresarial que deben estar en estrecha interrelación, para asegurar que la dinámica organizacional gire en torno de lo correcto. Son ellos la seguridad de la información, los aspectos económicos y financieros para la sostenibilidad, la gestión de los riesgos, y otros que se entrecruzan con los sistemas mencionados y que fortalecen y dan foco al quehacer de las organizaciones. Estos son los sistemas que la organización determina para asegurar que desde el momento de la planificación se puedan desarrollar los procesos que son cruciales para los resultados, y que éstos se realizan de forma controlada.

Dentro de los sistemas de gestión se consideran los que tienen que ver con la planificación de las finanzas, la determinación de presupuestos, el control de presupuestos, la ejecución, el control sobre los costos y los gastos, para asegurar la eficiencia del sistema productivo y la eficacia para alcanzar los objetivos planificados. Se incluye aquí también el sistema que define y controla las inversiones del negocio.

Otro de los factores determinantes dentro del enfoque de la gestión integral y dentro del componente sistemas de gestión es el que se relaciona con la gestión humana, que permite encontrar y desarrollar el talento de los seres humanos requerido por las organizaciones para generar ventajas competitivas. El conocimiento, las competencias y las capacidades que las personas deben desarrollar a partir de planes y programas bien estructurados para beneficio del quehacer de las organizaciones es determinante en el concepto de gestión integral que venimos construyendo.

Todos los sistemas de gestión, especialmente aquellos que han sido normalizados por medio de consensos na- cionales o internacionales, tienen elementos comunes, que resultan compatibles entre sí, y también elementos complementarios, específicos de su campo. Es posible al momento de implementar en una organización dos 0 más de dichos sistemas de gestión, hacerlo de forma que no se incurra en redundancias inoficiosas y que los elementos comunes se articulen debidamente para buscar resultados eficaces y eficientes; esto implica efectuar un análisis previo en aspectos como: relación con la planificación estratégica, metas, estructura organizacional, recursos, operacionalización, evaluación de resultados, acciones de mejora entre otros. Al resultado se le suele denominar como sistemas integrados de gestión o también sistemas de gestión integrada y es solo uno de los elementos de la gestión integral interna.

\section{Cultura de la organización}

La cultura se concibe como aquellas formas de sentir, pensar y actuar por parte de los seres humanos que componen las organizaciones, que se van consolidando a través del tiempo y de alguna manera son aprendidas por todos sus miembros y determinan en gran medida su comportamiento y sus actuaciones.

En ese contexto, los principios y valores con que "se vive" la organización son formadores fuertes de la cultura de la empresa y el concepto imperante de comunicación en la organización, así como los procesos que se tengan para este efecto, son de alto impacto en la construcción, desarrollo y fortalecimiento de las culturas organizacionales ${ }^{14}$.

Las culturas organizacionales son de alto impacto en la conducción de las empresas. Son las interrelaciones

14 Utilizamos la expresión "culturas organizacionales"- en plural porque la experiencia y las investigaciones han demostrado que en las organizaciones como sistemas sociales complejos, se reconoce más de un tipo de cultura. Culturas que están en relación con los niveles de pensamiento que en ellas se dan: pensamiento estratégico, pensamiento ejecucional y pensamiento operativo. 
de estas culturas las que hacen que la organización sea particularmente diferente a otras de su mismo medio, las que facilitan u obstaculizan el desarrollo de las estrategias, planes y objetivos que las organizaciones desde sus niveles de pensamiento estratégico han definido como importantes para llevar a cabo.

Los cuerpos gerenciales deben reconocer y conocer qué tipo de culturas son las que se viven en la organización para que pueda convertirlas en un factor a favor, para trabajar con eficiencia y eficacia en el logro de planes y propósitos establecidos y claramente comunicados. Y no, como comúnmente ocurre, que por falta de su involucramiento y buena comunicación se tornen en oponentes de los planes y objetivos de la organización.

En la Figura 1 se ilustra la forma en que se debe concebir la gestión integral en las organizaciones desde la perspectiva interna. Todos los anillos que representan los componentes de este modelo tienen como núcleo fundamental los resultados, que están directamente relacionados con las mujeres y hombres que las conforman, con el conocimiento que de ellos proviene para fortalecer la capacidad de las operaciones, controles y usos de recursos, mejoras de procesos, que permitan a la organización focalizarse en lo correcto, con flexibilidad, como antes se mencionó.

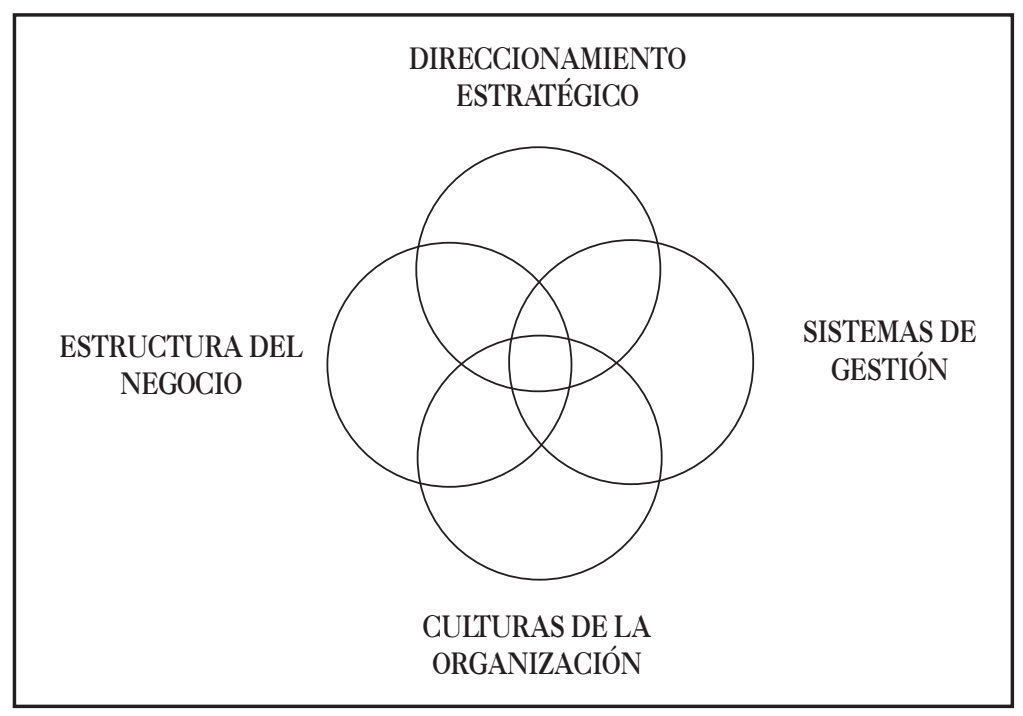

Figura 1. Componentes de la gestión integral interna. Fuente: los autores.

La gestión integral interna de las organizaciones se enfoca, entonces, en la comprensión de la organización desde las interacciones y dinámicas que se desarrollan con la articulación de la estructura del negocio, el direccionamiento estratégico, las culturas de la organización y la integración de los diferentes sistemas de gestión (normalizados o no) que la organización ha dispuesto para implementar su estrategia.

\section{El desarrollo sostenible}

El crecimiento económico desmesurado, resultante de la forma de pensar y de actuar que generó en el siglo XIX la Revolución Industrial, tiene unos costos ambientales y sociales que se empezaron a hacer evidentes sólo a mediados de los años sesenta del siglo $\mathrm{XX}$, y que generaron un movimiento creciente de pre- 
ocupación y de estudio por parte de las sociedades de los países desarrollados y de la Organización de las $\mathrm{Na}$ ciones Unidas; esta conciencia se acrecentó en parte porque muchos de esos efectos nocivos no se limitan al lugar donde se originan sino que tienen alcance global, como sucede con el calentamiento climático, la pobreza o las crisis financieras.

En la Declaración final de la Conferencia de la ONU sobre Medio Ambiente y Desarrollo celebrada en el año de 1992 en Río de Janeiro se formalizó el concepto de "desarrollo sostenible" a partir del Informe que la Comisión Brundtland había preparado para la ONU en 1987; en el Principio 3 de dicha declaración se establece que "El derecho al desarrollo se debe ejercer equitativamente de modo que se satisfagan las necesidades ambientales y de desarrollo de las generaciones presentes y de las futuras".

La interpretación que se ha dado al ámbito en que debe desenvolverse este principio es que se deben conciliar tres pilares básicos: el crecimiento económico, la cohesión social y la protección del medio ambiente; la responsabilidad por conciliar estos pilares recae tanto sobre los gobiernos como sobre las personas y también sobre los actores que, en forma visible y desde los inicios de la revolución industrial, han venido enfatizando el crecimiento económico, es decir, las organizaciones.

Las profundas interacciones entre los tres pilares aún no han sido totalmente dilucidadas y se prestan para una amplia variedad de interpretaciones, dependiendo de los intereses de quienes participan activamente en el tema (gobiernos de países desarrollados o de países en vía de desarrollo; compañías trasnacionales; comunidades afectadas; organizaciones no gubernamentales), por lo que se puede afirmar que el desarrollo sostenible es un campo que se encuentra todavía en plena construcción
Lo que se ha logrado aclarar es que las actividades humanas afectan el medio ambiente en formas diversas, básicamente a través de la extracción y explotación de los recursos naturales, muchos de ellos no renovables, los cuales son necesarios para promover el crecimiento económico y el bienestar de las personas (queda por definir cuáles serían los niveles deseables de bienestar); además, los desechos que generan las actividades humanas e industriales contaminan el medio ambiente y afectan la calidad de vida de las personas y de otras especies.

Por las anteriores razones el crecimiento económico debe ser viable, es decir, de acuerdo con la disponibilidad de los recursos naturales y la capacidad del medio ambiente para absorber los residuos y desechos. La preservación de los recursos naturales depende del número de personas que habitan el planeta y de sus hábitos de consumo, ya que el medio ambiente debe soportar esos niveles de utilización y de contaminación.

Algunos efectos no deseables de los fenómenos económicos globales pueden conducir a la fragmentación o la polarización de la sociedad por el aumento en las brechas sociales debido a fenómenos como la exclusión, la extrema inequidad en la distribución de la riqueza, el debilitamiento de la legitimidad de los gobiernos y la corrupción. Trabajar por la cohesión social implica entonces que se fomente la inclusión social y se generen espacios para promover el sentido de pertenencia de las poblaciones.

Bajo la premisa del desarrollo sostenible las organizaciones deben no sólo orientarse a maximizar las utilidades y beneficios económicos, la cual ha sido hasta ahora su meta esencial, sino también deben preocuparse por los aspectos ambientales y sociales de sus actividades productivas. 


\section{Gestión integral en las orǵanizaciones}

La gestión integral de las organizaciones, consideradas como sistemas abiertos, guarda relación tanto con los aspectos internos definidos anteriormente como con su contribución al desarrollo sostenible. En la Figura 2 se plantea dicha interacción.

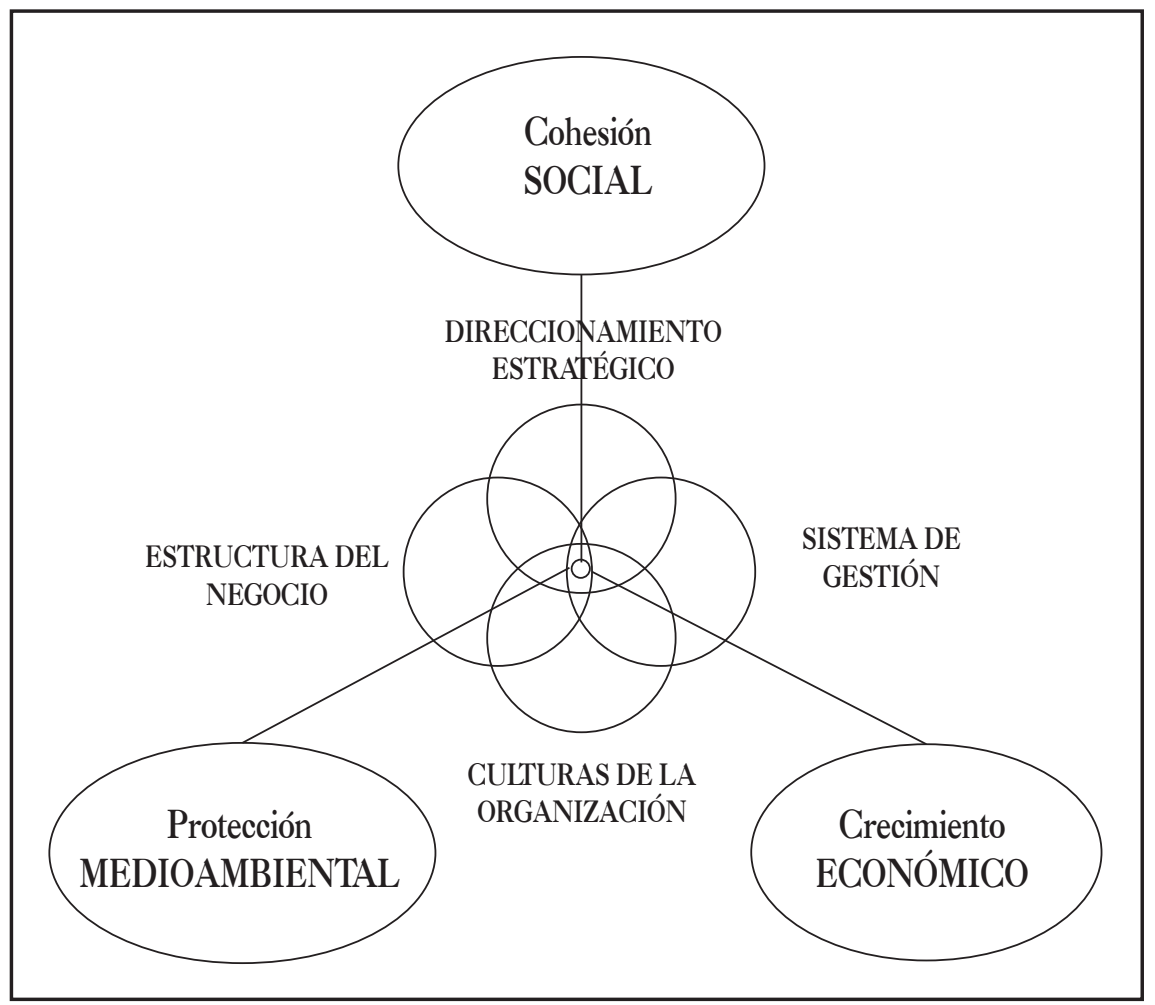

Figura 2. Gestión Integral de la organización. Fuente: los autores.

Las exigencias del mercado y de los clientes en cuanto a expectativas y requisitos, así como las demandas de la sociedad en aspectos macroeconómicos, de manejo equilibrado y sostenible de los recursos ambientales y sobre todo el efecto positivo que deberian tener las operaciones de negocios sobre el bienestar y la cohesión de la sociedad, se entrelazan con el ordenamiento interno de las organizaciones y sus capacidades (competencias internas).

La organización debe gestionarse integralmente de manera coherente, sistémica y sistemáticamente para responder a estos retos. La ocupación de los cuerpos gerenciales debe estar centrada en entender la integralidad de los componentes de la organización, para focalizar y gestionar su integralidad y no sólo unos pocos componentes de manera desarticulada. La gestión de la gerencia debe, entonces, articular alrededor de los tres pilares del desarrollo sostenible (que por supuesto se engloban dentro de una visión sistémica), mercados, competencia, clientes, productos, necesidades, requisitos, cultura del medio, por mencionar sólo algunos, con toda la capacidad interna de la organización, para hacerse reconocible por parte de mercados, clientes y usuarios en el largo plazo, y ser así sostenible. 
Por lo tanto, la búsqueda de soluciones teóricas y prácticas a la integración de los diferentes sistemas de gestión normalizados, de la gestión integral interna y de ésta con los pilares del desarrollo sostenible es el reto al cual deben responder las organizaciones modernas, sobre todo si tenemos en cuenta los turbulentos momentos por los cuales atraviesa la economía mundial.

Este es el desafío que asume la Maestría en Calidad y Gestión Integral: propiciar un mayor nivel competitivo para las organizaciones colombianas y latinoamericanas por medio de la reflexión, la propuesta de modelos y teorías, la validación de las mismas en la realidad de nuestras organizaciones y la discusión y diseminación del conocimiento en el campo de la gestión integral aquí planteada, de tal manera que pueda producir un movimiento en los niveles de pensamiento de la gerencia para generar modelos propios de conducción y gobierno en las organizaciones.

\section{REFERENCIAS}

CAMISÓN, César, et al. Gestión de la Calidad: Conceptos., enfoques, modelos y sistemas. Madrid: Pearson Prentice Hall. 2006. p. 145 - 147.
CONVENIO UNIVERSIDAD SANTO TOMÁS ICONTEC. Documento del Programa. 2006.

DÁVILA, Carlos. Teorías organizacionales y Administración. Bogotá: Mc Graw Hill. 2001.

ISHIKAWA, Kaoru. ¿Qué es el control total de la calidad? Bogotá: Norma. 1986. p.41-196

ICONTEC. Norma técnica colombiana NTC-ISO 9000:2005 Sistemas de gestión de la calidad. Fundamentos y vocabulario (numeral 3.1.1). Bogotá. 2005.

ORGANIZACIÓN DE LAS NACIONES UNIDAS. Declaración de Río sobre Medio ambiente y Desarrollo. Consultada el 28 de agosto de 2009 en http://www.un.org/documents/ga/conf151/ aconf15126-1annex1.htm,

TEJADA L., Fabio. El sistema de gestión de la calidad como aporte para el desarrollo de habilidades y capacidad gerencial en las Pymes de Bogotá. Revista SIGNOS: Investigación en sistemas de gestión, junio de 2009, vol. 1, no.1, p. 33-68. 